\title{
Exploring Multispectral Iris Recognition beyond 900nm
}

\author{
Arun Ross, Raghunandan Pasula, Lawrence Hornak
}

\begin{abstract}
Most iris recognition systems acquire images of the eye in the $700 \mathrm{~nm}-900 \mathrm{~nm}$ range of the electromagnetic spectrum. In this work, the iris is examined at wavelengths beyond $900 \mathrm{~nm}$. The purpose is to understand the iris structure at longer wavelengths and to determine the possibility of performing cross-spectral iris matching. An acquisition system is first designed for imaging the iris at narrow spectral bands in the $950 \mathrm{~nm}-1650 \mathrm{~nm}$ range. Next, the left and right images of the iris are acquired from 25 subjects in order to conduct the analysis. Finally, the possibility of performing cross-spectral matching and multispectral fusion at the match score level is investigated. Experimental results suggest: (a) the feasibility of acquiring iris images in wavelengths beyond $900 \mathrm{~nm}$ using InGaAs detectors; (b) the possibility of observing different structures in the iris anatomy at various wavelengths; and (c) the potential of performing cross-spectral matching and multispectral fusion for enhanced iris recognition.
\end{abstract}

\section{INTRODUCTION}

The rich texture of the iris coupled with the apparent stability of its structure render it a useful biometric. The textural content of the iris is characterized by numerous structures, both fibrous and cellular, contained on its anterior surface including ligaments, crypts, furrows, collarette, moles, freckles, etc. The "individuality" of each iris is assumed to be a consequence of the random morphogenesis ${ }^{1}$ of its textural relief. From an image processing perspective, the stochastic nature of this texture can be described by applying a Gabor filter bank on an iris image and examining the ensuing phasor response. The phasor response at each pixel is quantized using two bits of information and the resulting IrisCode is used for encoding and matching iris images [1][2]. Traditional iris recognition systems use the near-infrared (NIR) spectrum in the 700-900nm wavelength to capture the iris, since longer wavelengths can penetrate dark-colored irides, thereby eliciting their texture which cannot be easily observed in the visible spectrum. The effect of melanin, the major color inducing compound, is negligible at longer wavelengths; this ensures that the observed information is predominantly due to the texture of the iris rather than its pigmentation (see Fig. 1).

The use of multispectral information to enhance recognition performance has been explored in the case of several biometric traits including fingerprint [6], face [7][8], hand [9], and iris [3]. Multispectral analysis involves examining the images of an object at multiple spectral bands. In the context of iris, Boyce et al. [3] used information from the

The authors are with Lane Department of Computer Science and Electrical Engineering at West Virginia University. arun.ross@mail.wvu.edu, rpasula@mix.wvu.edu,

lawrence. hornak@mail.wvu. edu

${ }^{1}$ http://www.cl.cam.ac.uk/ jgd1000/anatomy.html

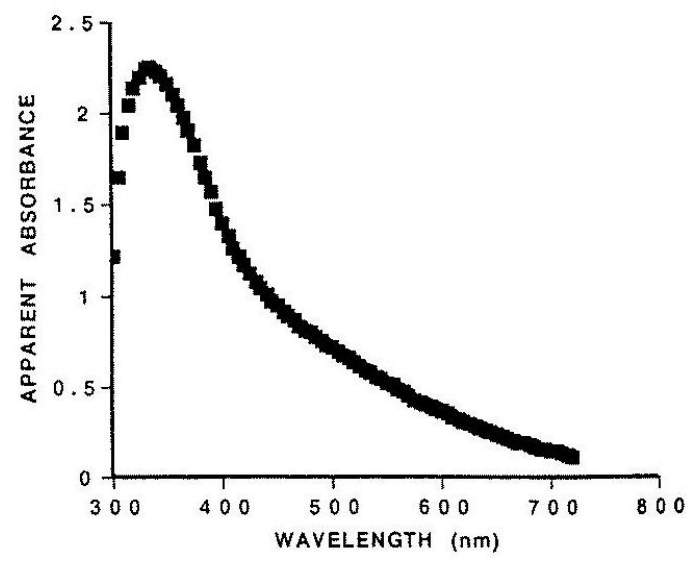

Fig. 1. Absorption spectrum of melanin. The effect of melanin at longer wavelengths is negligible. Taken from [5].

Red, Green and Blue bands of the visible spectrum along with information from the near IR band to improve the segmentation process; further, they demonstrated the feasibility of performing multispectral fusion at the score level to improve the recognition accuracy of an iris system. Park and Kang [10] used multispectral information to determine the authenticity of the iris being imaged by the system. Further, they fused the multispectral images into a single grayscale image which was subsequently used in the encoding and matching phases. This work explores the possibility of using iris information from images obtained beyond the wavelength of $900 \mathrm{~nm}$, i.e., beyond those wavelengths traditionally used. The eventual goal is to better understand the structure of the iris and to improve recognition performance. This represents the first attempt in the literature to analyze the iris at these wavelengths. This paper is organized as follows. Goal and objectives are presented in section II. The image acquisition system is described in section III. The images acquired using this system are analyzed in section IV. The pre processing schemes applied to the images, and the encoding and matching techniques used to generate the results are discussed in section V. The results are presented and analyzed in section VI. Summary and Conclusions are presented in section VII.

\section{GOAL AND OBJECTIVES}

The goal of this work is to understand the structure and composition of iris at multiple wavelengths by examining its spectrum beyond $900 \mathrm{~nm}$ and up to $1700 \mathrm{~nm}$ as shown in Fig. 2. Such a study is essential for various reasons:

a) In certain tactical environments (e.g., a warfighter scenario), the availability of multiple physical detectors (i.e., 


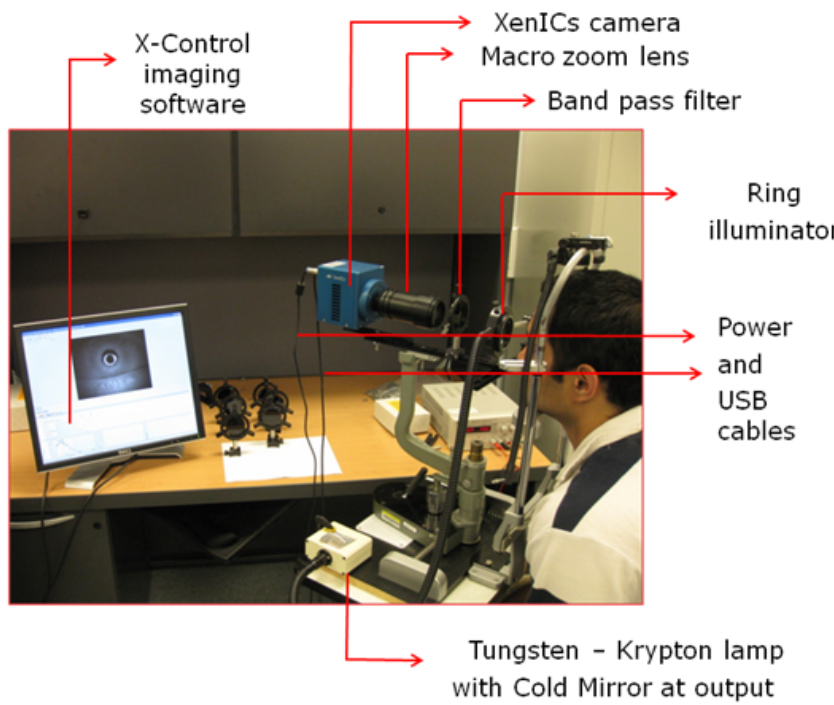

Fig. 2. Image acquisition setup.

sensors) may facilitate the acquisition of biometric information at longer wavelengths. To understand the utility of such images, a detailed analysis of the iris texture at these wavelengths is necessary.

b) If the structure of the iris is observed to differ across multiple spectral bands, then fusing this information may be beneficial for iris recognition. For example, such a scheme may be used to enhance the "individuality" of the iris biometric.

c) Anti-spoofing methods can be developed by studying the response of structures within the live iris at different wavelengths.

The conjecture here is that the iris structure produces a differential response based on the spectral band under consideration and, hence, diverse textural information can be obtained at multiple spectral bands. This property is exploited in this work to perform score level fusion across spectral bands to improve recognition performance. Further, the possibility of matching iris images across spectral bands (i.e., cross-spectral matching) is studied.

\section{IMAGE ACQUISITION SYSTEM}

\section{A. Imaging system design}

One of the most challenging aspects of this research is the design of the image acquisition system. The image acquisition setup is shown in Fig. 2. A XenIC XEVA-818 camera served as the imager for this study. The camera has an Indium Gallium Arsenide (InGaAs) 320 x 256 Focal Plane Array (FPA) with $30 \mu \mathrm{m}$ pixel pitch, 98\% pixel operability and three stage thermoelectric cooling. The XEVA- 818 has a relatively uniform spectral response from $950-1700 \mathrm{~nm}$ wavelength (the Short Wavelength Infra-Red (SWIR) band) across which the InGaAs FPA has largely uniform quantum efficiency (see Fig. 3). Response falls rapidly at wavelengths lower than $950 \mathrm{~nm}$ and near $1700 \mathrm{~nm}$.
Given the objective of this study to compare and understand the iris images across multiple spectral bands within the SWIR band, the acquisition system was designed to illuminate the iris with broadband illumination from a single source that spanned the spectral range of interest. Images of the reflected light from the iris were then acquired through a series of spectral windows in this broad band through the use of a series of narrow band filters at the camera input.

The setup to achieve this acquisition is shown in Fig. 2. A Tungsten-Krypton DC light source is used as the broadband source to illuminate the eye. The $300-2200$ $\mathrm{nm}$ wavelength output of the source is relatively flat over the camera's spectral response range, decreasing towards longer wavelengths. The broadband output of this source is cut with a cold mirror to exclude visible wavelengths below $750 \mathrm{~nm}$ for the comfort of the subject and to promote pupil dilation. The broadband light is then delivered to the eye using a ring illuminator with bandpass sufficient for the experiment's spectral range. The broadband luminance levels for the experimental setup described here conformed with ANSI/IESNA RP-27.1-05 for near infrared exposure limits under the condition of weak aversion stimulus. The reflected light from the subject's eye is then collected through the center of the ring light, through a bandpass filter and imaged by the XEVA- 818 camera using a conventional macro zoom lens.

Eight 100 nm FWHM, 4-cavity nonpolarizing band pass filters centered at 950, 1050, 1150, 1250, 1350, 1450, 1550, and $1650 \mathrm{~nm}$ were fabricated to specification by Andover Corporation and used in sequence to image a subject's eye in spectral slices across the SWIR band under the aforementioned broadband illumination. Peak transmission of these filters on Borofloat glass substrates ranged from 87 percent at $950 \mathrm{~nm}$ to 74 percent at $1650 \mathrm{~nm}$. Refer to Fig. 3. A similar optical transmission efficiency through the macro zoom lens is expected at these wavelengths. As a result, as low as 50 percent transmission to the camera's FPA can be expected at the experiment's longest wavelengths.

\section{B. Image Acquisition Protocol}

Five images each of the left and right eyes of a subject were taken using 950,1050, 1150, 1250, 1350, 1450, 1550 and $1650 \mathrm{~nm}$ band pass filters, respectively. A small dataset containing samples from 25 subjects was used in this study. Sample multispectral iris images pertaining to a single eye are shown in Fig. 4.

The five images, associated with a single band-pass filter, correspond to images taken at five intervals of time, a few seconds apart, where subjects are asked to remove their head from the chin-rest and pan their head either side before resuming the collection process. The image focus was manually adjusted each time an image was taken.

\section{IMAGE ANALYSIS}

In this section, the images acquired using the aforementioned process are analyzed. 


\section{A. Variations in Contrast and Average Brightness}

It is observed that there are variations in image contrast and average brightness across the spectral bands as shown in Fig. 5. This can partially be explained by the water absorption $^{2}$ of the aqueous humour, a thick watery region between the lens and cornea. The water absorption spectrum indicates that absorption is very high at around 1400nm and this is believed to result in the relatively "dark" images obtained using the 1450nm band pass filter. A similar observation can be made for images acquired using the $1650 \mathrm{~nm}$ band pass filter.

However, this does not completely explain the photometric attributes of these multi spectral images. For example, if the explanation above based on water absorption were to be the sole reason for the "dark" images, then the samples obtained using the $1550 \mathrm{~nm}$ band pass filter would be expected to be darker than the images taken using the 1450nm band pass filter; but the images at $1550 \mathrm{~nm}$ filter are observed to be a little "brighter", as can be observed in the histogram plot shown in Fig. 5(g). This indicates the possibility of other contributing factors, such as the different absorption properties of individual components of the iris structure, to the overall brightness and contrast. It must be noted that the average power incident on the eye varied as a function of the wavelength used. This could have also impacted the quality of the images procured at different bands, particularly the images beyond $1450 \mathrm{~nm}$.

\section{B. Differential response of iris}

Different components of the iris seem to respond differently at multiple spectral bands for dark colored irides. One such prominent observation is the presence of a blurred limbic boundary at $950 \mathrm{~nm}$ and a sharp limbic boundary at $1350 \mathrm{~nm}$. So, images at $1350 \mathrm{~nm}$ could potentially be used for segmentation (due to the prominent limbic edges) while

${ }^{2}$ http://www.lsbu.ac.uk/water/vibrat.html

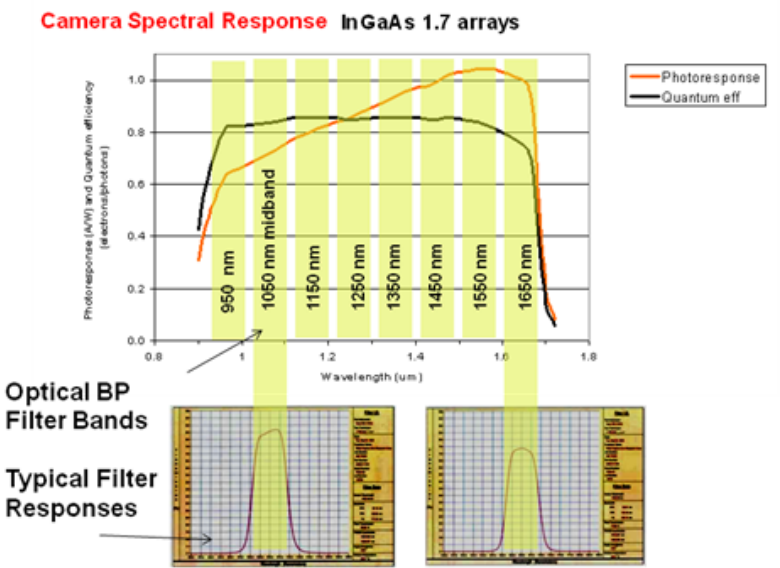

Fig. 3. Camera photo response and its quantum efficiency shown along with the filter response of band pass filters used in the experiment. Iris images are obtained in 100nm spectral bands. Image courtesy XenIC and Andover Corporation.

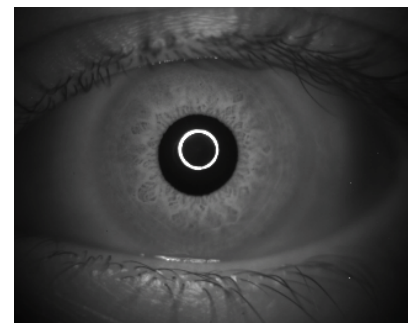

(a)

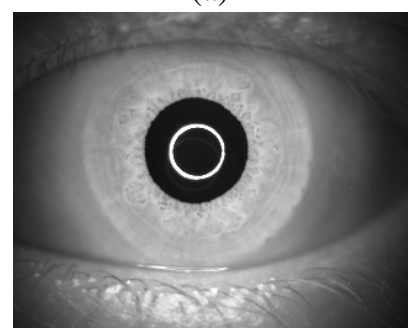

(c)

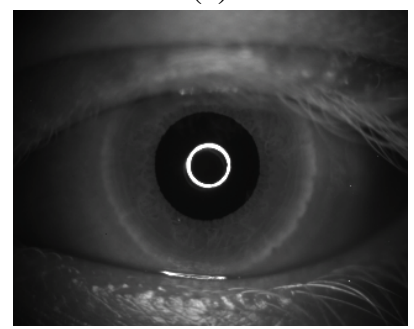

(e)

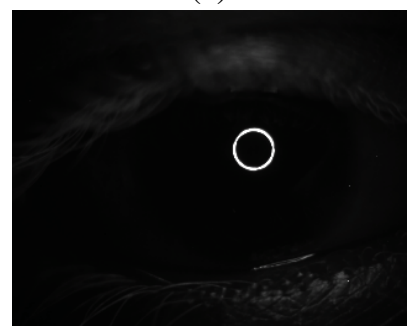

(g)

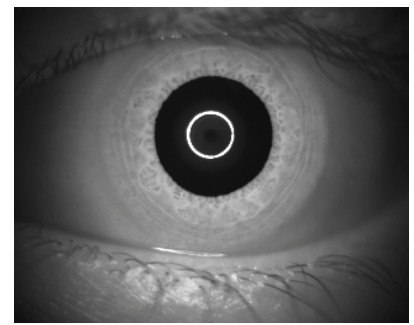

(b)

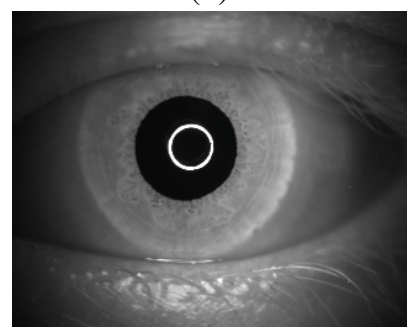

(d)

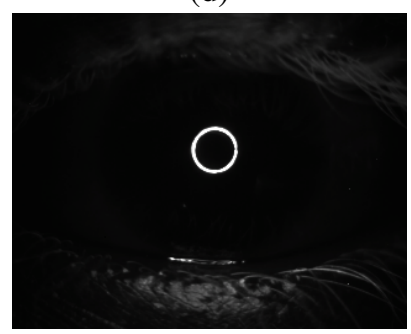

(f)

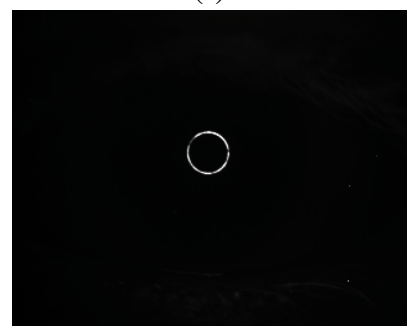

(h)
Fig. 4. Sample images obtained at wavelengths (a) $950 \mathrm{~nm}$, (b) $1050 \mathrm{~nm}$, (c) $1150 \mathrm{~nm}$, (d) $1250 \mathrm{~nm}$, (e) $1350 \mathrm{~nm}$, (f) $1450 \mathrm{~nm}$, (g) $1550 \mathrm{~nm}$, and (h) $1650 \mathrm{~nm}$.

the images taken at shorter wavelengths could be used for extracting the iris texture.

Also, eyelashes that are observed to be dark at $950 \mathrm{~nm}$, appear grey at $1350 \mathrm{~nm}$ and white beyond 1350nm (Fig. 6). Thus, if these multispectral images were obtained simultaneously (i.e., if they are co-registered), then segmentation could be significantly improved due to the differential response of the various ocular structures at different spectral bands.

Even after applying enhancement techniques like adaptive histogram equalization, it was observed that the textural information of the iris varied across the different spectral bands. The images after applying adaptive histogram equalization are shown in Fig. 7.

\section{Improper illumination and focus}

Although the focus was manually adjusted each time an image was acquired, there are a few out-of-focus images 


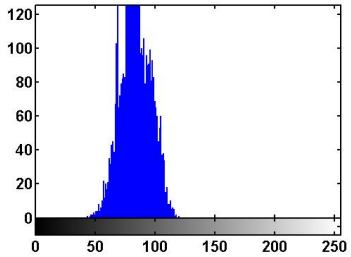

(a)

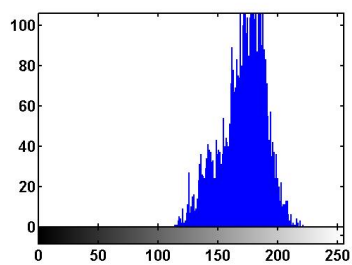

(c)

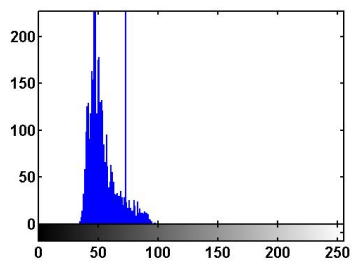

(e)

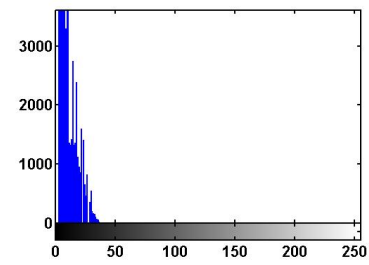

$(\mathrm{g})$

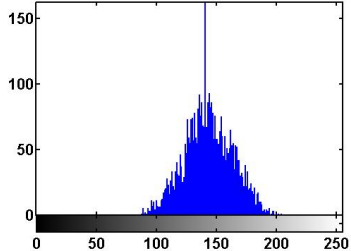

(b)

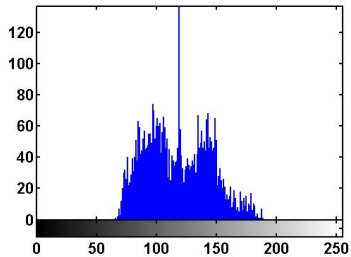

(d)

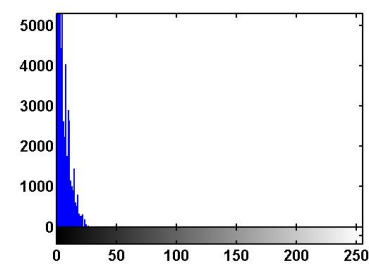

(f)

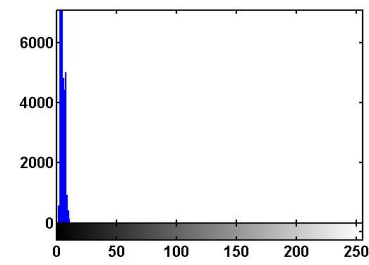

(h)
Fig. 5. Histograms of normalized irises for the images shown in Fig. 4: (a) $950 \mathrm{~nm}$, (b) $1050 \mathrm{~nm}$, (c) $1150 \mathrm{~nm}$, (d) $1250 \mathrm{~nm}$, (e) $1350 \mathrm{~nm}$, (f) $1450 \mathrm{~nm}$, (g) $1550 \mathrm{~nm}$, and (h) $1650 \mathrm{~nm}$. The contrast and average intensity changes with wavelength.

due to the movement of the subject's head on the chinrest. Similarly, as the ring illuminator was manually adjusted, identical illumination across all subjects is not guaranteed. However, these factors do not drastically alter the structure of the iris image since, for the most part, utmost care is taken to impose uniformity in the data collection process across subjects.

\section{PREPROCESSING AND MATCHING}

\section{A. Segmentation}

In order to decouple the effect of inaccurate segmentation on the performance of iris recognition, the irides in the acquired images were manually segmented. The pupil boundary and limbic boundary were assumed to be circular although they are not usually concentric [1]. Noisy regions including eyelashes and occlusions were manually marked in the image as shown in Fig. 8. This ensured that segmentation was not a confounding factor for further investigation.

\section{B. Normalization, Pre-processing and Encoding}

Daugman's rubber sheet model [1] was used to unwrap the iris from Cartesian coordinates to a pseudo-polar coordinate system. The mask denoting the segmented iris was also unwrapped into this new coordinate system. The normalized image was subject to adaptive histogram equalization as part of pre-processing - see Fig. 8. Adaptive histogram equalization improves the contrast of the image by transforming the grayscale values of an image using contrast-limited adaptive histogram equalization (CLAHE). This algorithm stretches the histogram of local group of pixels, called tiles, rather than stretching the histogram of the entire image (Fig. 9). This ensures that the overall histogram shape of the entire image is not overly modified. This also ensures that not much noise is induced in the grayscale values of the image. Each row of the normalized iris is considered as a 1-D signal and $\log$ Gabor filters are applied to this signal resulting in a complex valued output.

The frequency response of a Log-Gabor filter is given as;

$$
G(f)=\exp \left[\frac{-\left(\log \left(f / f_{o}\right)\right)^{2}}{2\left(\log \left(\sigma / f_{o}\right)\right)^{2}}\right]
$$

where $f_{o}$ represents the center frequency, and $\sigma$ denotes the bandwidth of the filter. The output of the filter is phase quantized to four levels (with 0's and 1's) using Daugman's method. This binary feature vector is referred to as the IrisCode. Note that each row corresponds to a circular ring on the iris and maximum independence occurs in the angular direction, i.e., along the columns in the pseudo-polar coordinate system. Masek's code [4] was modified to process the obtained images and also used to normalize, encode and match the iris images.

\section{Matching}

In order to generate a match score, the Hamming distance between two iris codes was computed after taking into account the masked bits corresponding to noise due to eyelashes, eyelids, occlusion, etc. The Hamming distance between two iris codes, code $\mathrm{A}$ and codeB, with corresponding mask arrays, maskA and maskB, is given as [1]:

$$
H D=\frac{\|(\operatorname{code} A \otimes \operatorname{code} B) \bigcap \operatorname{mask} A \bigcap \operatorname{mask} B\|}{\|\operatorname{mask} A \bigcap \operatorname{mask} B\|}
$$

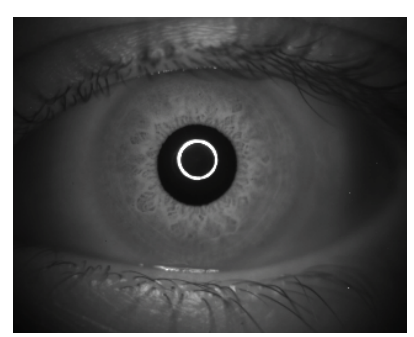

(a)

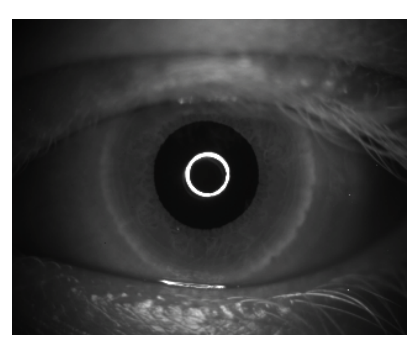

(b)
Fig. 6. Images taken at wavelengths (a) $950 \mathrm{~nm}$ and (b) $1350 \mathrm{~nm}$. The color of eyelashes is black at $950 \mathrm{~nm}$ where as they turn white at $1350 \mathrm{~nm}$ and beyond. Also note the sharp limbic boundary at $1350 \mathrm{~nm}$. 


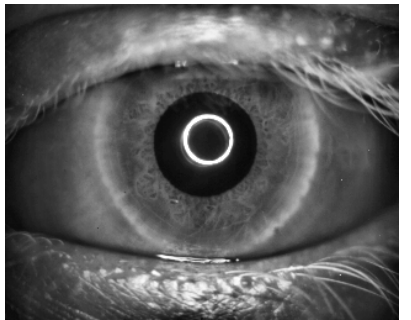

(a)

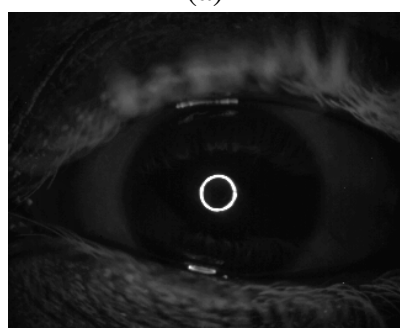

(c)

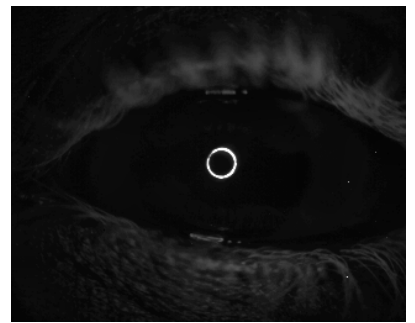

(b)

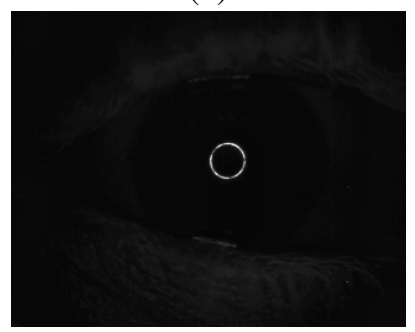

(d)
Fig. 7. Adaptive histogram equalized images corresponding to (a) $1350 \mathrm{~nm}$, (b) $1450 \mathrm{~nm}$, (c) $1550 \mathrm{~nm}$ and (d) $1650 \mathrm{~nm}$ wavelengths. No distinct texture is available at these wavelengths. Note the whitened eyelashes.

\section{EXPERIMENTAL RESULTS}

\section{A. Data set}

A dataset containing samples from 25 subjects was used to conduct the following study. Only five spectral bands corresponding to $950,1050,1150,1250$ and 1350nm were used in the experiment as the textural clarity of the images from the remaining bands was too low to be of benefit for this study. More research is required in order to establish the utility of these bands (i.e., 1450, 1550 and 1650nm) in iris recognition and to determine if the texture can be reliably extracted.

\section{B. Definitions}

Intra-spectral genuine scores are obtained when two samples from the same spectral band of the same eye of a subject are matched. Cross-spectral genuine scores are obtained when an image from one spectral band is matched against an image from a different spectral band, where both the images correspond to the same eye of the same subject. Intra-spectral impostor scores are obtained when an image of, say, the left eye of a subject from a particular spectral band is matched against an image of the left eye of a different subject from the same spectral band.

\section{Fusion}

Since 5 images corresponding to multiple spectral bands are available for each (probe) eye, the match scores generated by comparing these individual images against their counterparts in the database (gallery) can be fused. Fusion is expected to benefit the recognition process in the following ways: (a) when images pertaining to a subset of the spectral bands are not of good quality, then images from the other bands could be used to perform matching; (b) if all the images are corrupted by noise, then fusion at the score level

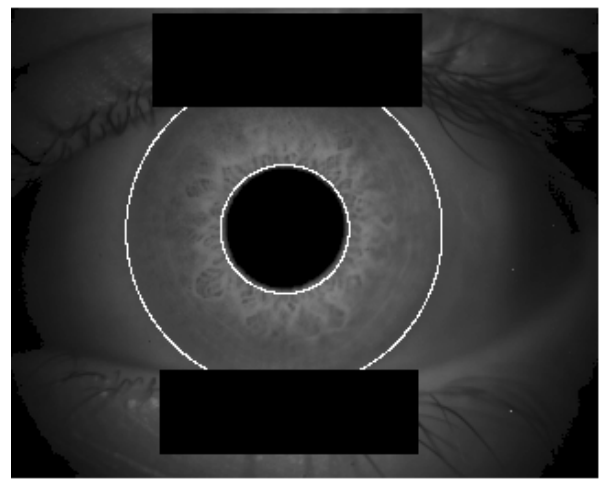

(a)

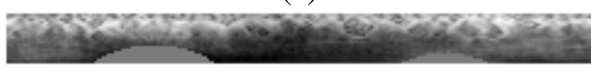

(b)

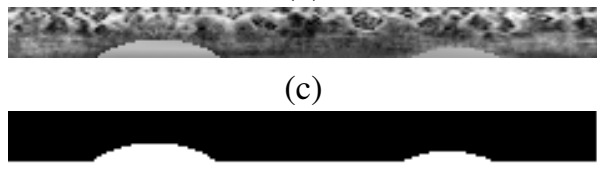

(d)

Fig. 8. (a) Manually segmented iris image, obtained at 950nm, showing the masked pixels as black; (b) Unwrapped iris; (c) Adaptive histogram equalized image of image in (b); and (d) Mask array showing whitened regions corresponding to noise.

can help reduce the variance associated with the noise. In this work, fusion is accomplished using the simple sum rule.

In the current acquisition system, the multispectral images of a single iris are not acquired simultaneously due to the fundamental limitation of the configuration of the system. However, future advances in optics and imaging can make this possible thereby exploiting the benefits of parallel fusion.

\section{Histogram plots}

A total of 2,500 intra-spectral genuine scores; 25,000 cross-spectral genuine scores; and 75,000 intra-spectral impostor scores are generated. The normalized histogram plots of distance scores for each spectrum band (for all 25 subjects, the plot includes the scores for both left and right eyes of a subject), along with the normalized histogram plot of the scores from all the bands, are shown in Fig. 10. The histogram plot of fused scores is shown in Fig. 11.

The intra-spectral impostor scores are observed to be fairly well separated from the intra-spectral genuine scores. Crossspectral genuine scores, on the other hand, are spread over a wide range of scores. Further, their modes are shifted to the right of that of intra-spectral genuine scores. In some cases, the cross-spectral genuine scores overlap with the intra-spectral impostor scores. This is expected because of the varying textural information across the spectral bands of a single eye. Most of the overlap is due to images taken at $1350 \mathrm{~nm}$ because of their low textural quality. However, the experiments confirm the possibility of performing crossspectral matching beyond $900 \mathrm{~nm}$. Also, a few outliers for genuine intra-spectral scores can be observed in the histogram plot for 950nm, shown in Fig. 10(a). This may be due to improper segmentation by the human operator as 
the limbic boundary was blurred in some eye images making it difficult for the operator to accurately segment the image. It is clearly evident that fusion is beneficial and that the fused genuine and impostor scores are better separated than the scores before fusion.

\section{E. Box Plots}

Fig. 12 shows the box plots for genuine intra-spectral and genuine cross-spectral scores. It is observed that genuine intra-spectral scores have the least median value and are

Before

adaptive histogram equalization

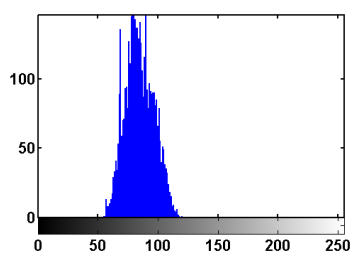

(a)

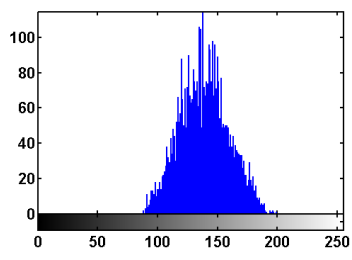

(b)

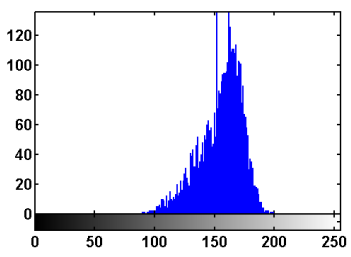

(c)

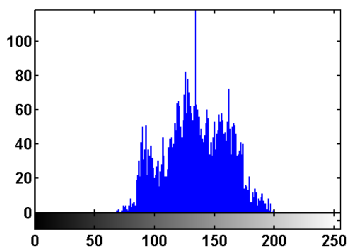

(d)
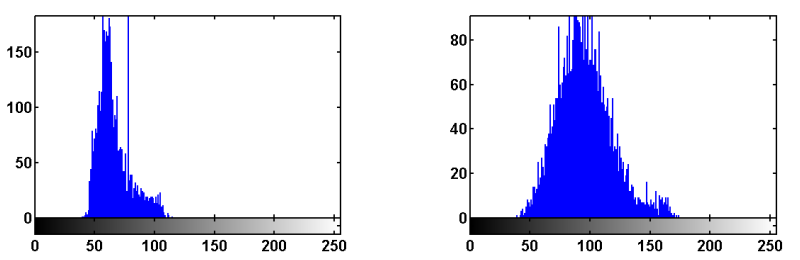

(e)

Fig. 9. Histogram plots of images taken at (a) $950 \mathrm{~nm}$, (b) $1050 \mathrm{~nm}$, (c) 1150 , (d) $1250 \mathrm{~nm}$ and (e) $1350 \mathrm{~nm}$, before and after adaptive histogram equalization is applied. Contrast variation, spread in the horizontal direction, is improved after adaptive histogram equalization. mostly spread around this value. Another observation is that the distribution of genuine intra-spectral scores is almost similar across all the spectral bands, as can be visualized in the box plots along the main diagonal. Also, when comparing images of the same iris at two spectral bands that are farther apart, the distance scores tend to be higher (i.e., worse) as can be observed from the first row of plots in Fig. 12. The distribution of scores tends to "move up" in the box plots when traversing from left to right in this row.

\section{SUMMARY AND CONCLUSIONS}

The purpose of this work was to explore the feasibility of conducting iris recognition beyond $900 \mathrm{~nm}$. It represents the first attempt in the literature to do so. Such an analysis is required to understand the structure of the iris that is revealed at longer wavelengths. Further, in tactical environments, the use of detectors/sensors beyond the visible range would necessitate the processing of biometric images acquired at longer wavelengths. To this end, an acquisition system using an $\mathrm{InGaAs}$ focal plane array camera was designed to acquire a small data set of images in the $900 \mathrm{~nm}-1700 \mathrm{~nm}$ range. Initial experiments suggest the possibility of cross-spectral matching in the 900 to $1400 \mathrm{~nm}$ range. However, more detailed experiments are necessary to confirm this possibility for irides corresponding to different eye colors.

From a computer vision perspective, the difference in iris texture across spectral bands is an intricate function of the physical characteristics of the detector (i.e., sensor) and the anatomical differences in the iris structure revealed in these bands. This justifies the use of a fusion scheme to enhance recognition accuracy as was borne out in the experiments.

Currently, an automated segmentation algorithm that can localize the iris accurately at multiple wavelengths is being developed [11]. Better enhancement techniques for photometric normalization and geometric registration may be required to improve the performance of multispectral matching in large datasets [12]. Fusion at the image level and the feature level are also being investigated [13]. Finally, the design of a layered model of the iris is being explored to account for variations in intensity and contrast as a function of individual spectral bands [14].

\section{ACKNOWLEDGMENT}

This work was supported in part by the NSF Center for Identification Technology Research (CITeR) at West Virginia University. The authors thank Peter Hein for assistance with the data collection activity. Thanks to the volunteers who provided ocular data for the analysis conducted in this work. Finally, thanks to the anonymous reviewers who provided valuable comments and suggestions.

\section{REFERENCES}

[1] J. Daugman, "High confidence visual recognition of persons by a test of statistical independence", IEEE Transactions on Pattern Analysis and Machine Intelligence, Vol. 15, No. 11, pp. 1148-1161, 1993.

[2] K. W. Bowyer, K. Hollingsworth, and P. J. Flynn, "Image understanding for iris biometrics: A survey", Computer Vision and Image Understanding, Vol. 110, Issue 2, pp. 281 - 307, May. 2008. 


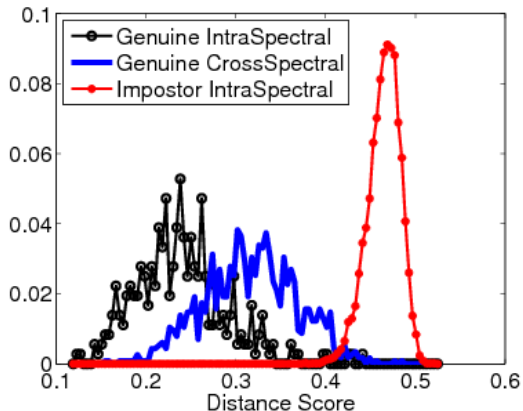

(a)

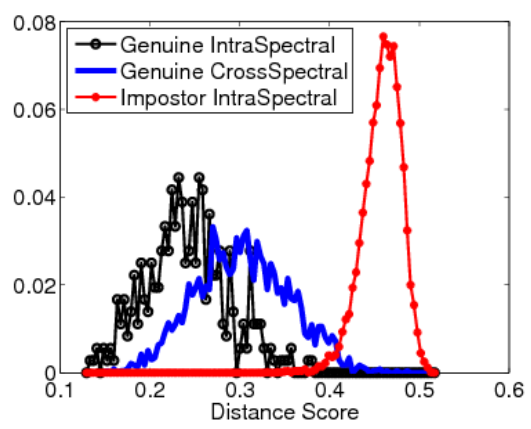

(d)

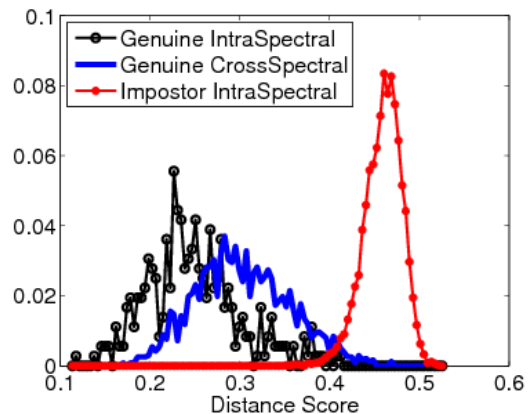

(b)

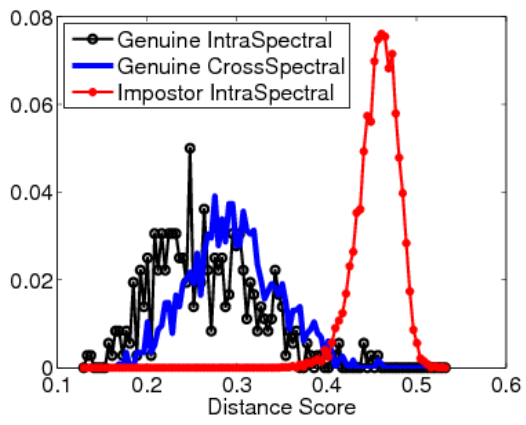

(e)

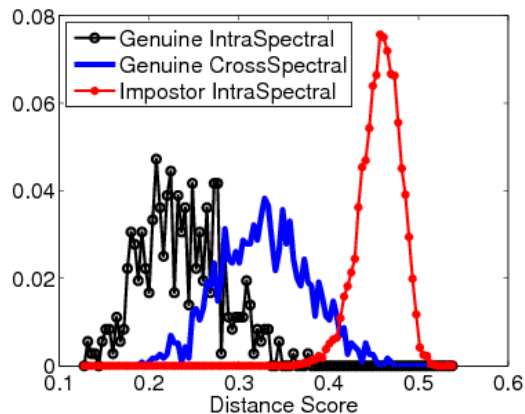

(c)

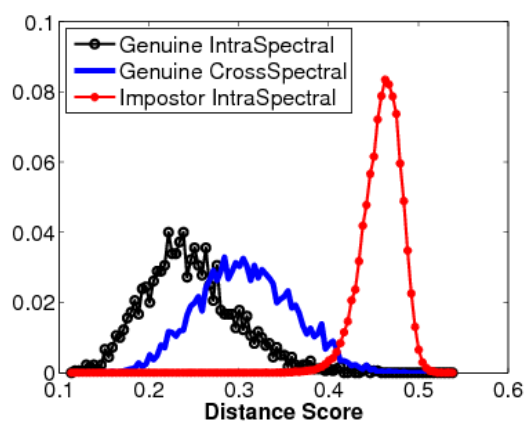

(f)

Fig. 10. Normalized histogram plots of genuine cross-spectral (blue dotted line), genuine intra-spectral (black line) and impostor intra-spectral (red line with markers) distance scores for (a) $950 \mathrm{~nm}$, (b) $1050 \mathrm{~nm}$, (c) $1150 \mathrm{~nm}$, (d) $1250 \mathrm{~nm}$, (e) 1350nm and (f) all the wavelengths combined.

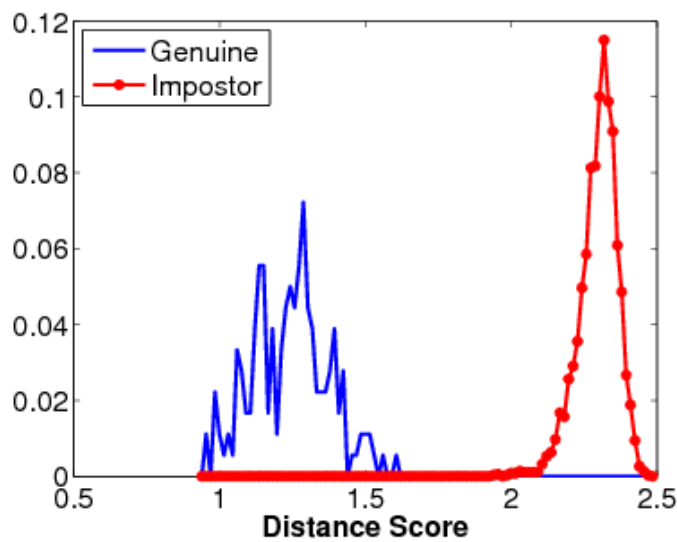

Fig. 11. The normalized histogram plot showing the result of fusing intraspectral scores across the five spectral bands $(950 \mathrm{~nm}, 1050 \mathrm{~nm}, 1150 \mathrm{~nm}$, $1250 \mathrm{~nm}, 1350 \mathrm{~nm})$. Fusion using the simple sum rule results in good separation between genuine scores and impostor scores.

[3] C. Boyce, A, Ross, M. Monaco, L. Hornak and X. Li, "Multispectral Iris Analysis: A Preliminary Study", IEEE Computer Society Workshop on Biometrics at the Computer Vision and Pattern Recognition Conference, June 2006.

[4] L. Masek, "Recognition of Human Iris Patterns for Biometric Identification", BSE Dissertation, University of Western Australia, 2003.

[5] N. Kollias, "The spectroscopy of human melanin pigmentation". In:
L. Zeise, M. R. Chedekel, T. B. Fitzpatrick (eds), Melanin: Its Role in Human Photoprotection, pp. 31 - 38, Valdenmar Publishing, 1995.

[6] R. Rowe and K. Nixon, "Fingerprint enhancement using a multispectral sensor", Proc. of the SPIE Biometric Technology for Human Identification II, Volume 5779, pp. 81-93, Orlando, March 2005.

[7] Z. Pan, G. Healey, M. Prasad and B. Tromberg, "Face recognition in hyperspectral images", IEEE Transactions on Pattern Analysis and Machine Intelligence, Vol. 12, No. 12, pp. 1552-1560, December 2003.

[8] D. A. Socolinsky, "Multispectral face recognition". In: A. K. Jain, P. J. Flynn and A. Ross (eds), Handbook of Biometrics, pp. 293-313, 2007.

[9] R. K. Rowe, U. Uludag, M. Demirkus, S. Parthasaradhi and A. K. Jain, "A Multispectral Whole-hand Biometric Authentication System", Proceedings of Biometric Symposium (BSYM), Baltimore, September 2007.

[10] J. Park and M. Kang, "Multispectral iris authentication system against counterfeit attack using gradient-based image fusion," Optical Engineering, Vol. 46, November 2007.

[11] S. Shah and A. Ross, "Iris Segmentation Using Geodesic Active Contours," IEEE Transactions on Information Forensics and Security (TIFS), 2009 (to appear).

[12] M. Vatsa, R. Singh and A. Noore, "Improving Iris Recognition Performance using Segmentation, Quality Enhancement, Match Score Fusion and Indexing," IEEE Transactions on Systems, Man, and CyberneticsB, Vol. 38, No. 4, pp. 1021-1035, 2008.

[13] A. Ross, "An Introduction to Multibiometrics," Proc. of the 15th European Signal Processing Conference (EUSIPCO), Poznan, September 2007.

[14] G. Franois, P. Gautron, G. Breton, K. Bouatouch, "Image-Based Modeling of the Human Eye," IEEE Transactions on Visualization and Computer Graphics, Vol. 15, No. 5, pp. 815-827, September/October 2009. 


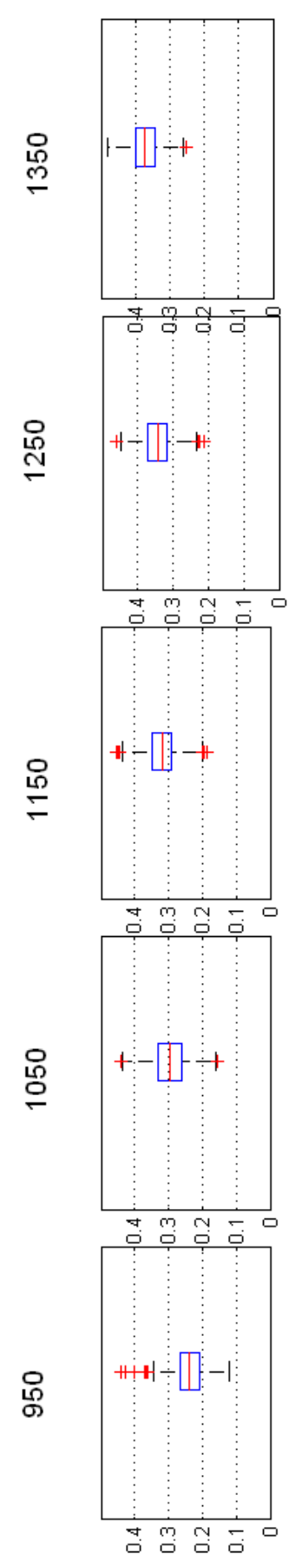

용
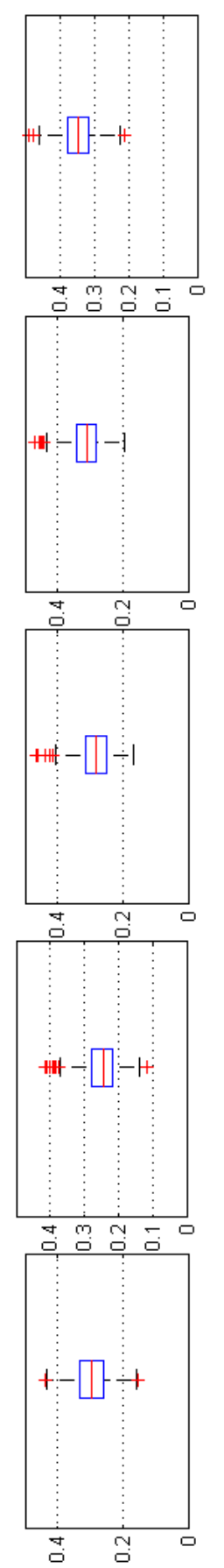

융
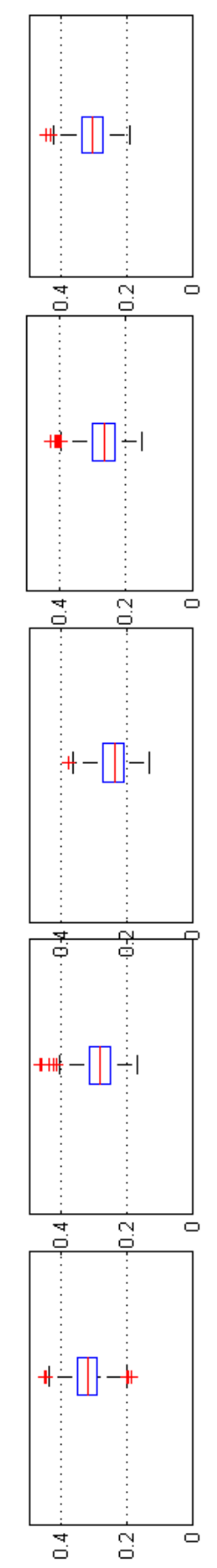

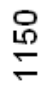
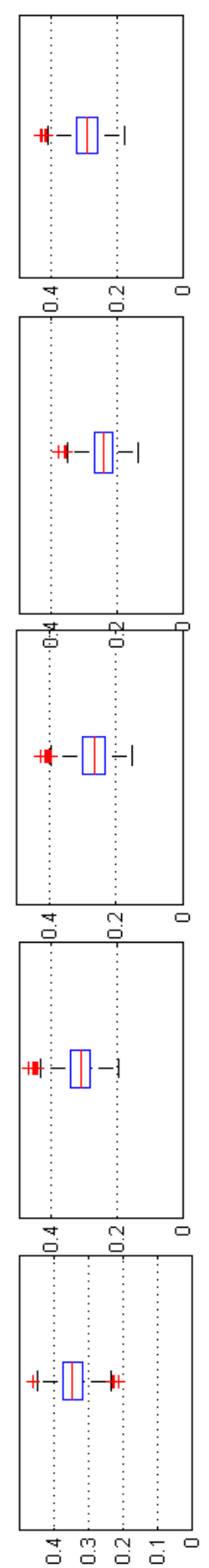

怘 